\title{
Recent progress in CVD diamond detector R\&D
}

The RD42 Collaboration

R. Wallny ${ }^{* 24}$ A. Alexopoulos, ${ }^{3}$ M. Artuso, ${ }^{20}$ F. Bachmair, ${ }^{24}$ L. Bäni, ${ }^{24}$ M. Bartosik, ${ }^{3}$ H. Beck, ${ }^{23}$ V. Bellini, ${ }^{2}$ V. Belyaev, ${ }^{12}$ B. Bentele, ${ }^{19}$ A. Bes, ${ }^{27}$ J-M. Brom, ${ }^{7}$ M. Bruzzi, ${ }^{4}$ G. Chiodini, ${ }^{26}$ D. Chren, ${ }^{18}$ V. Cindro, ${ }^{9}$ G. Claus, ${ }^{7}$ J. Collot, ${ }^{27}$ J. Cumalat, ${ }^{19}$ A. Dabrowski, ${ }^{3}$ R. D'Alessandro, ${ }^{4}$ D. Dauvergne, ${ }^{27}$ W. de Boer, ${ }^{10}$ C. Dorfer,${ }^{24}$ M. Dünser, ${ }^{3}$ G. Eigen, ${ }^{30}$ V. Eremin, ${ }^{6}$ G. Forcolin, ${ }^{22}$ J. Forneris, ${ }^{15}$ L. Gallin-Martel, ${ }^{27}$ M-L. Gallin-Martel, ${ }^{27}$ K. K. Gan, ${ }^{13}$ M. Gastal, ${ }^{3}$ M. Goffe, ${ }^{7}$ J. Goldstein, ${ }^{17}$ A. Golubev, ${ }^{8}$ A. Gorišek, ${ }^{9}$ E. Grigoriev, ${ }^{8}$ J. Grosse-Knetter, ${ }^{23}$ A. Grummer, ${ }^{21}$ M. Guthoff, ${ }^{3}$ B. Hiti, ${ }^{9}$ D. Hits, ${ }^{24}$ M. Hoeferkamp, ${ }^{21}$ T. Hofmann, ${ }^{3}$ J. Hosselet, ${ }^{7}$ F. Hügging, ${ }^{1}$ C. Hutton, ${ }^{17}$ J. Janssen, ${ }^{1}$ H. Kagan, ${ }^{13}$ K. Kanxheri, ${ }^{28}$ R. Kass, ${ }^{13}$ M. Kis, ${ }^{5}$ G. Kramberger, ${ }^{9}$ S. Kuleshov, ${ }^{8}$ A. Lacoste, ${ }^{27}$ S. Lagomarsino, ${ }^{4}$ A. Lo Giudice, ${ }^{15}$ I. Lopez Paz, ${ }^{22}$ E. Lukosi, ${ }^{25}$ C. Maazouzi, ${ }^{7}$ I. Mandić, ${ }^{9}$ C. Mathieu, ${ }^{7}$ M. Menichelli, ${ }^{28}$ M. Mikuž, ${ }^{9}$ A. Morozzi, ${ }^{28}$ J. Moss, ${ }^{29}$ R. Mountain, ${ }^{20}$ A. Oh, ${ }^{22}$ P. Olivero, ${ }^{15}$ D. Passeri, ${ }^{28}$ H. Pernegger, ${ }^{3}$ R. Perrino, ${ }^{26}$ F. Picollo, ${ }^{15}$ M. Pomorski, ${ }^{11}$ R. Potenza, ${ }^{2}$ A. Quadt, ${ }^{23}$ F. Rarbi, ${ }^{27}$ A. Re,${ }^{15}$ M. Reichmann, ${ }^{24}$ S. Roe, ${ }^{3}$ D. A. Sanz Becerra, ${ }^{24}$ M. Scaringella, ${ }^{4}$ C. J. Schmidt, ${ }^{5}$ S. Schnetzer, ${ }^{14}$ E. Schioppa, ${ }^{3}$ S. Sciortino, ${ }^{4}$ A. Scorzoni, ${ }^{28}$ S. Seidel, ${ }^{21}$ L. Servoli, ${ }^{28}$ D. S. Smith, ${ }^{13}$ B. Sopko, ${ }^{18}$ V. Sopko, ${ }^{18}$ S. Spagnolo, ${ }^{26}$ S. Spanier, ${ }^{25}$ K. Stenson, ${ }^{19}$ R. Stone, ${ }^{14}$ B. Stugu, ${ }^{30}$ C. Sutera, ${ }^{2}$ M. Traeger, ${ }^{5}$ W. Trischuk, ${ }^{16}$ M. Truccato, ${ }^{15}$ C. Tuve, ${ }^{2}$ J. Velthuis, ${ }^{17}$ N. Venturi, ${ }^{3}$ S. Wagner, ${ }^{19}$ J. C. Wang, ${ }^{20}$ N. Wermes, ${ }^{1}$ M. Yamouni, ${ }^{27}$ J. Zalieckas, ${ }^{30}$ and M. Zavrtanik ${ }^{9}$

${ }^{1}$ Universität Bonn, Bonn, Germany, ${ }^{2}$ INFN/University of Catania, Catania, Italy, ${ }^{3}$ CERN, Geneva, Switzerland, ${ }^{4}$ INFN/University of Florence, Florence, Italy, ${ }^{5}$ GSI, Darmstadt, Germany, ${ }^{6}$ Ioffe Institute, St. Petersburg, Russia, ${ }^{7}$ IPHC, Strasbourg, France, ${ }^{8}$ ITEP, Moscow, Russia, ${ }^{9}$ Jožef Stefan Institute, Ljubljana, Slovenia, ${ }^{10}$ Universität Karlsruhe, Karlsruhe, Germany, ${ }^{11}$ CEA-LIST Technologies Avancées, Saclay, France, ${ }^{12}$ MEPHI Institute, Moscow, Russia, ${ }^{13}$ The Ohio State University, Columbus, OH, USA, ${ }^{14}$ Rutgers University, Piscataway, NJ, USA, ${ }^{15}$ University of Torino, Torino, Italy, ${ }^{16}$ University of Toronto, Toronto, ON, Canada, ${ }^{17}$ University of Bristol, Bristol, UK, ${ }^{18}$ Czech Technical University, Prague, Czech Republic, ${ }^{19}$ University of Colorado, Boulder, CO, USA, ${ }^{20}$ Syracuse University, Syracuse, NY, USA, ${ }^{21}$ University of New Mexico, Albuquerque, NM, USA, ${ }^{22}$ University of Manchester, Manchester, UK, ${ }^{23}$ Universität Göttingen, Göttingen, Germany, ${ }^{24}$ ETH Zürich, Zürich, Switzerland, ${ }^{25}$ University of Tennessee, Knoxville, TN, USA, ${ }^{26}$ INFN-Lecce, Lecce, Italy, ${ }^{27}$ LPSC-Grenoble, Grenoble, France, ${ }^{28}$ INFN-Perugia, Perugia, Italy, ${ }^{29}$ California State University, Sacramento, CA, USA, ${ }^{30}$ University of Bergen, Bergen, Norway

E-mail: rainer.wallnyephys.ethz.ch

We present an overview of the latest developments from RD42 in diamond detector R\&D. They include the radiation hardness coefficients for $800 \mathrm{MeV}$ and $24 \mathrm{GeV}$ protons, the hit detection efficiency for two 3D detector prototypes with two different readout chips and a novel method for investigating charge transport in single crystal diamonds.

The 28th International Workshop on Vertex Detectors - Vertex2019,

13-18 October, 2019,

Lopud, Croatia

*Speaker. 


\section{Introduction}

Progress in experimental particle physics continues to depend crucially upon the ability to carry out experiments in a high radiation environment. The flux of highly energetic particles is strongest close to the interaction point. At the High Luminosity Large Hadron Collider (HL-LHC), in the best performance scenario, the radiation levels in the innermost layers of the CMS detector are projected to achieve $3.0 \times 10^{16} \mathrm{n}_{\mathrm{eq}} / \mathrm{cm}^{2}[1,2]$. The development of new materials and testing new device designs that are able to withstand such harsh radiation levels are most important for these applications. A particle sensor based on Chemical Vapor Deposition (CVD) diamond is a very attractive candidate for such an environment. Not only does diamond have a higher displacement energy than silicon, therefore promising higher radiation tolerance [3], but also, the large band gap allows for operation of diamond devices without any cooling, and as a consequence, significantly reduces the infrastructure in the inner layers of particle detectors. The RD42 collaboration [4] studies CVD diamond for usage in high energy physics experiments as tracking and beam monitoring devices. In this paper we present the latest results of RD42 collaboration in understanding the radiation hardness of CVD diamond sensors, show advances in 3D sensor technology that promise to extend the radiation tolerance to even higher levels, and discuss a novel method to investigate charge transport in single crystal CVD (scCVD) diamonds.

\section{Radiation tolerance}

The RD42 collaboration has recently updated the study of radiation damage in CVD diamonds by $800 \mathrm{MeV}$ and $24 \mathrm{GeV}$ protons [5]. A total of four samples, three polycrystalline CVD (pCVD) diamonds and one single crystal CVD diamond, were irradiated with $800 \mathrm{MeV}$ protons and a total of three samples, one pCVD diamond and two scCVD diamonds, were irradiated with $24 \mathrm{GeV}$ protons. Each diamond was irradiated in steps to various fluences. After each fluence the diamond was metallized with a strip metallization and connected to low noise multichannel readout. The pulse height in the devices, induced by a minimum ionizing particle (MIP), was measured for several bias voltages of both positive and negative polarity. The hit position of the MIP was predicted with $\sim 3 \mu \mathrm{m}$ precision by a beam telescope. Figure 1 shows the pulse height distributions in the fiducial area for negative bias voltage $(\sim 2 \mathrm{~V} / \mu \mathrm{m})$ for different fluences of $800 \mathrm{MeV}$ protons. The mean of each distribution is linearly proportional to the Charge Collection Distance (CCD), from which a schubweg $\lambda$ (an average distance a charge carrier travels before being immobilized) can be extracted using Equation 2.1.

$$
\frac{c c d}{t}=\sum_{i=e, h} \frac{\lambda_{i}}{t}\left[1-\frac{\lambda_{i}}{t}\left(1-e^{-\frac{t}{\lambda_{i}}}\right)\right]
$$

where $t$ is the thickness of the diamond.

The inverse schubweg versus fluence is fitted for each sample separately with Equation 2.2 as shown in the Figure 2.

$$
\frac{1}{\lambda}=\frac{1}{\lambda_{0}}+\kappa \phi
$$

where $\lambda_{0}$ the initial schubweg before the irradiation, $\kappa$ the damage constant and $\phi$ is the fluence [6]. 


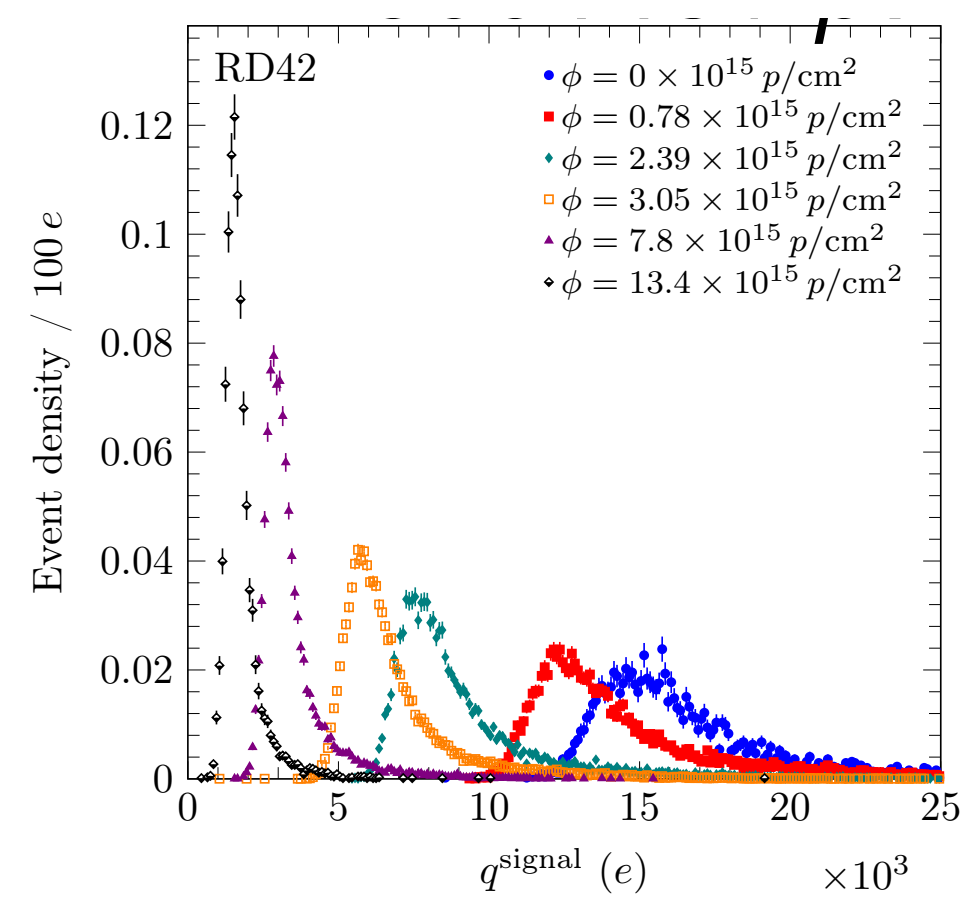

Figure 1: Pulse height distributions for negative bias voltage after various fluences of $800 \mathrm{MeV}$ protons [5].

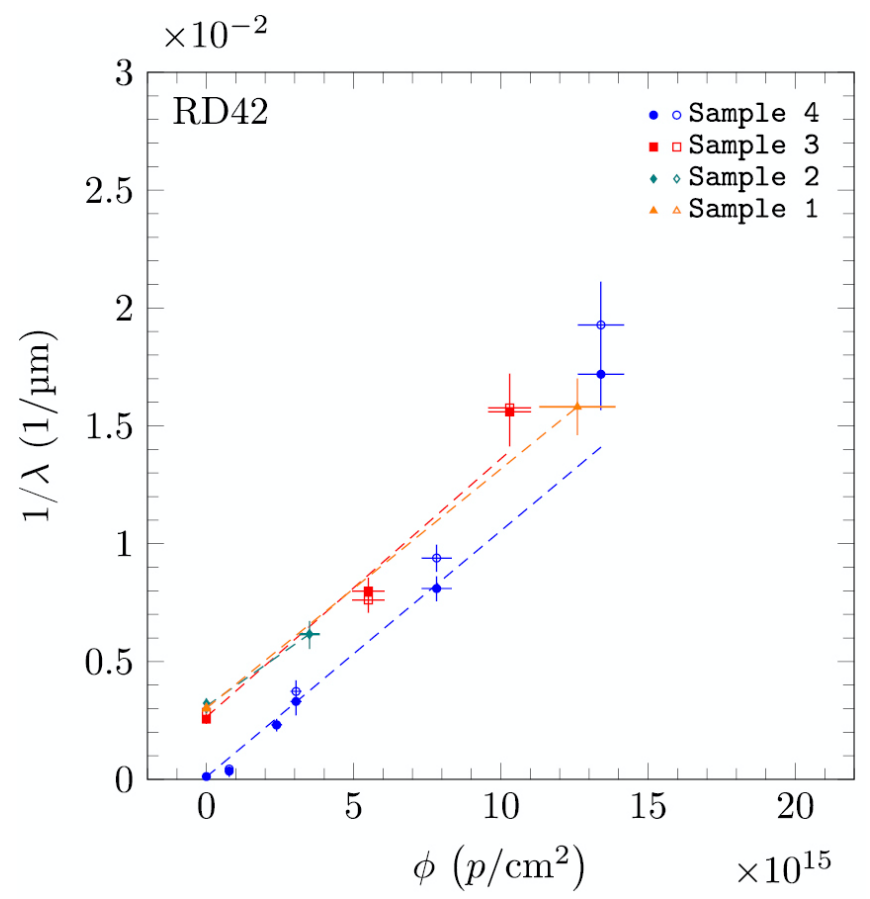

Figure 2: Schubweg versus fluence of $800 \mathrm{MeV}$ for three $\mathrm{pCVD}$ diamonds and one scCVD diamond fitted individually with Equation 2.2 [5]. 
Observing that the change in the pulse height of all diamond samples due to irradiation by the same particle species at the same energy is found to be within one sigma of each other, we average the result. This observation suggests that the radiation damage in the different samples follows the same underlying physical mechanism of radiation. The resulting coefficient is the damage constant for diamond for a particular particle species at a particular energy. The damage coefficient for $24 \mathrm{GeV}$ protons is $0.62 \pm 0.07 \times 10^{-18} \mathrm{~cm}^{2} \mu \mathrm{m}^{-1}$. Dividing the coefficients for other particle species by the $24 \mathrm{GeV}$ proton damage constant we obtain the relative coefficients shown in Table 1 .

\begin{tabular}{l|l|l}
\hline Particle & Energy & Relative $\kappa$ \\
\hline Proton & $24 \mathrm{GeV}$ & 1.0 \\
\hline & $800 \mathrm{MeV}$ & $1.68 \pm 0.13$ \\
\hline & $70 \mathrm{MeV}$ & $2.6 \pm 0.4$ \\
\hline & $25 \mathrm{MeV}$ & $4.9 \pm 0.6$ \\
\hline Neutron & $\sim 1 \mathrm{MeV}$ & $4.9 \pm 0.5$ \\
\hline
\end{tabular}

Table 1: The relative damage constants for various particle species normalized to $24 \mathrm{GeV}$ proton equivalent $[5,7]$.

These relative damage coefficients can be used to normalize the fluences of other particle species to the equivalent fluence of the $24 \mathrm{GeV}$ protons.

\section{3D Pixel detectors}

The idea to put electrodes in the bulk of a sensor instead at the surface to bring them closer to each other and therefore reduce the drift distances of charged carriers (and the probability of being trapped before reaching the electrode) was introduced by S. Parker et al. in 1997 [8]. In diamond, the conductive electrodes in the bulk (albeit with a high resistance therefore we will call them resistive) are formed with the aid of a femtosecond laser. The material in the focal point of the laser converts from the insulating diamond phase into a mixture of various phases that includes graphite, which is conductive [9]. By moving the focal point linearly through the diamond the column electrode is formed. Four resistive electrodes arranged in a square pattern with one electrode in the middle of the square form a 3D cell. The electrode in the center of the square is the readout electrode while the electrodes in the corners of the square are the bias electrodes. Two arrays of approximately 4000 cells with the cell size of $50 \mu \mathrm{m} \times 50 \mu \mathrm{m}$ were fabricated in two pCVD diamonds. The efficiency of column electrode fabrication with a diameter of $\sim 2.6 \mu \mathrm{m}$ was $99.7 \%$. The readout column electrodes of one array were ganged together in a $1 \times 5$ pattern while the readout column electrodes of the other array were ganged together in a $2 \times 3$ pattern, in order to match the readout of FE-I4 [10] and PSI46digv2.1respin [11] readout chips, respectively, as shown in Figures $3 \mathrm{a}$ and $3 \mathrm{~b}$. The sensors were then bump bonded to the corresponding readout chips using two different methods. The sensor with the $1 \times 5$ pattern was bump bonded to an FE-I4 readout chip with Sn-Ag solder bumps. The sensor with the $2 \times 3$ pattern was bump bonded to a PSI46digv2.1respin readout chip using indium bumps. The indium bumps were deposited on both the readout chip and the sensor and were then brought into contact using only pressure, i.e. no melting of indium after the deposition was performed. Thus, the connection was formed by solid inter-diffusion of indium at room temperature. 


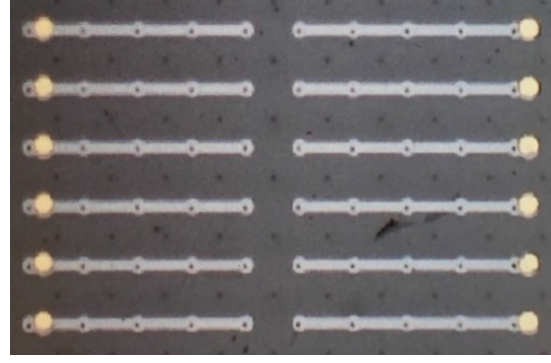

(a) $1 \times 5$

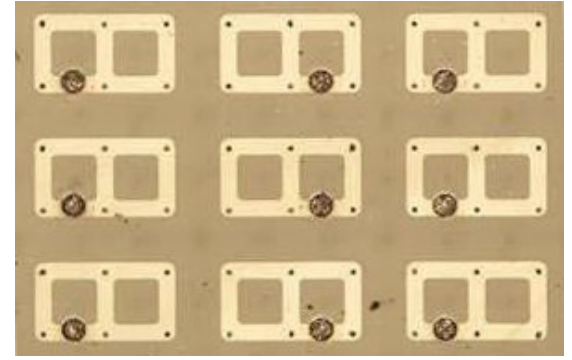

(b) $2 \times 3$

Figure 3: 3D diamond sensors with $50 \mu \mathrm{m} \times 50 \mu \mathrm{m}$ cells metallized for bump bonding to (left) FE-I4 chip and (right) PSI46digv2.1 respin chip.

Both devices were tested under similar conditions by minimum ionizing particles $(\sim 120 \mathrm{GeV}$ protons) at CERN's Super-Proton-Synchrotron test beam area H6A [12]. The data were analyzed with Beam Telescope Analysis (BTA) software [13]. The hit position of the particles on the detector were reconstructed with a precision of $\sim 5 \mu \mathrm{m}$ with a beam telescope. The efficiency of hit reconstruction was measured with respect to hit position (see Figure 4). The efficiency is visibly higher for the device with the $2 \times 3$ pattern (Figure $4 b$ ). We suspect that the reason for lower efficiency in the $1 \times 5$ pattern (Figure $4 \mathrm{a}$ ) was potentially related to the modifications introduced in the Sn-Ag bump bonding process. A few inefficient spots in the $2 \times 3$ pattern device are due to the broken readout electrodes in the sensor. This was also confirmed by a visual inspection of the column electrodes in the 3D sensor.

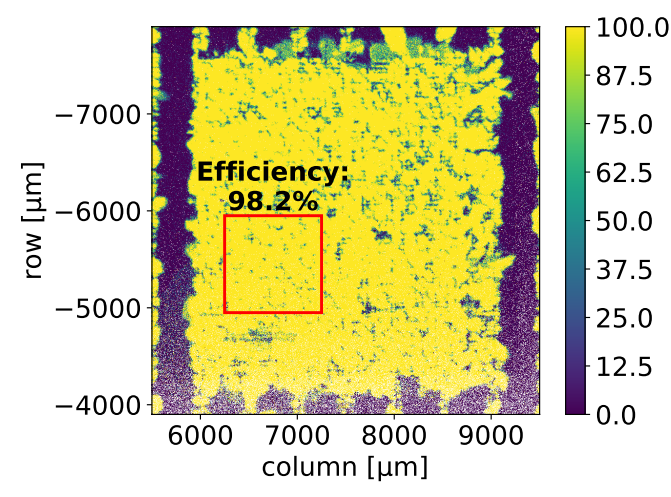

(a)

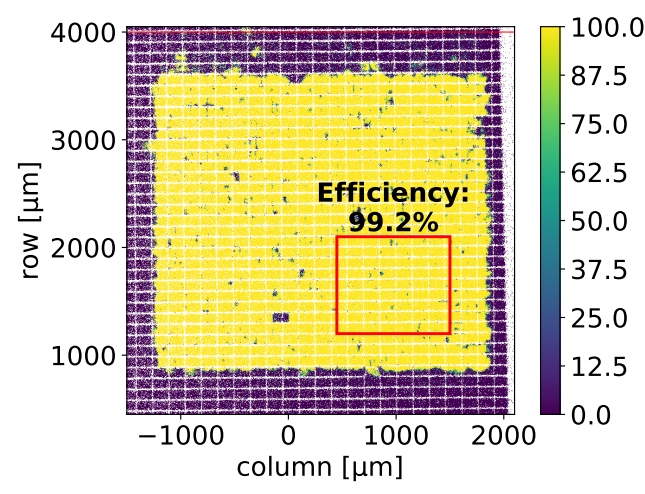

(b)

Figure 4: Efficiency versus hit position for detector readout with (left) FE-I4 chip and detector readout with (right) PSI46digv2.1respin chip.

The fiducial region, marked by a red rectangle in the plot of Figure 4, was chosen in order to avoid the known defects in both the 3D sensor and the readout chip. The efficiency versus bias voltage (Figure 5) in the fiducial region already saturates for both devices at $20 \mathrm{~V}$ and reaches $98.2 \%$ for the $1 \times 5$ pattern device and $99.2 \%$ for $2 \times 3$ pattern device. The $0.4 \%$ inefficiency in the $2 \times 3$ pattern device is due to the tracks passing through either one of the column electrodes. The remaining $0.4 \%$ is not understood and may be due to the low field region at the saddle points of 
electric field distribution. Such low field regions are inherent in the 3D sensor design and therefore expected. However, further analysis is required to confirm or reject whether this hypothesis explains the observed inefficiency pattern. At any rate, these results, combined with the ability to

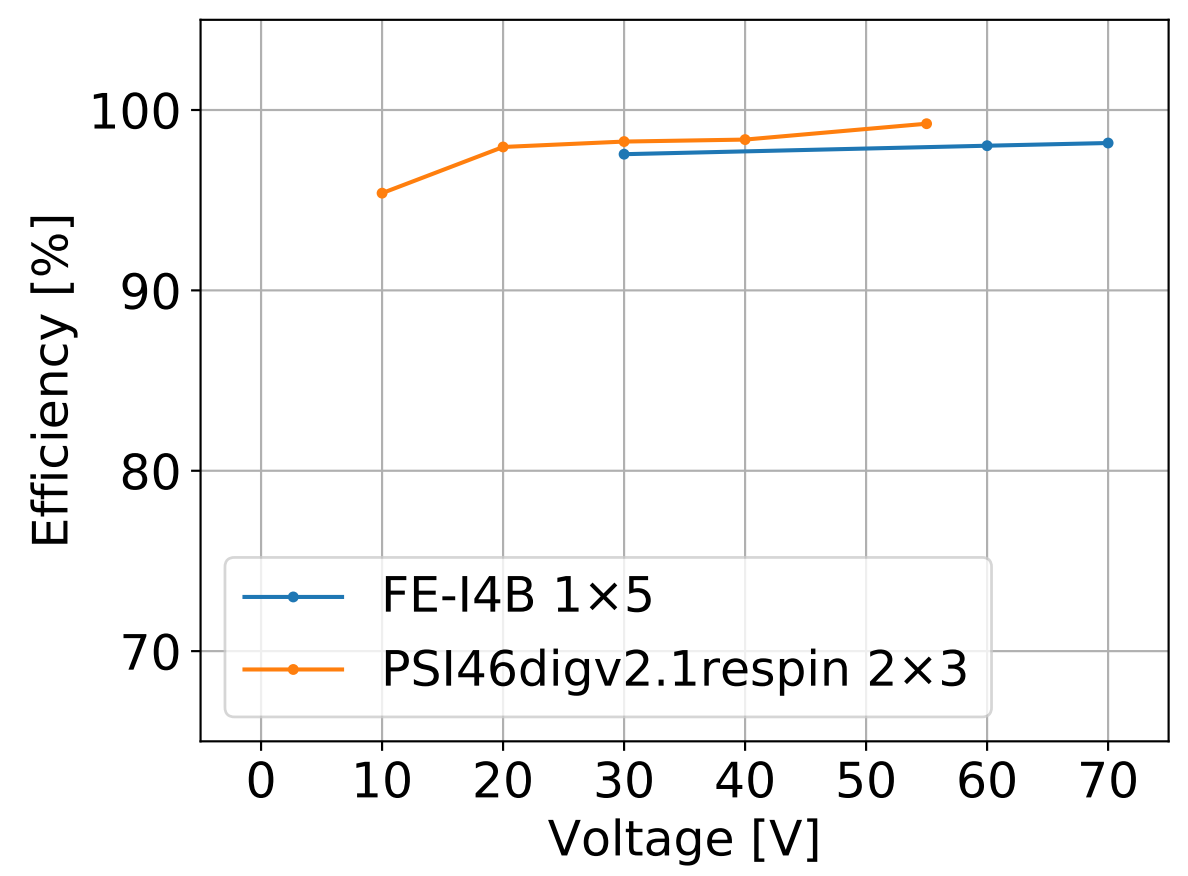

Figure 5: Efficiency versus bias for detector readout with FE-I4 chip (blue) and detector readout with PSI46digv2.1respin chip (orange).

operate without any cooling, make such a device an attractive alternative to the tracking devices based on silicon. Further research is still required to confirm the same high efficiency remains after irradiation, as well as to scale up the size of the devices and to improve yield of bulk electrodes.

\section{3D charge transport mapping by the edge-TCT method}

One way to understand the peculiarities in the behaviour of diamond sensors is to study the charge transport in diamond. The Transient Current Technique (TCT), whereby a charge in the sensor is generated by a laser beam entering through the edge of the device (edge-TCT), is an interesting method for the study of charge transport in scCVD diamonds [14]. This method allows to infer such parameters as the charge trapping rates, electric field distribution and, through it, space charge distribution. In diamond, due to a large bandgap, the electric charge generation by a laser pulse with a 400-nm long wavelength occurs through a two-photon absorption [15]. This effect has a further advantage; the charge generation is limited to the focal point of the laser beam, where the density of photons is the highest. Thus, edge-TCT allows for 3D profiling of the electric field in a diamond device.

The setup, described in detail in [15], consists of a femtosecond laser generating 800-nm 100 -fs long photon bunches. The frequency of photons is doubled by a BBO (Barium Borate) crystal and the laser beam is then focused in the diamond sample. The diamond sample consists 
of a diamond plate $\sim 540 \mu \mathrm{m}$ thick and approximately $4.6 \mathrm{~mm} \times 4.6 \mathrm{~mm}$ in area, with contact pads deposited on each side of the plate. The edges of the contact pads are about 200-300 $\mu \mathrm{m}$ away from the edge of the diamond. The charge carriers, generated in the focal point, travel in the electric field in the diamond producing a current pulse that is amplified by a $40 \mathrm{~dB}$ broad band amplifier and recorded by an oscilloscope. A few samples of such pulses are shown in Figure 6. Since the bias on the top electrode is negative, the current pulses, originating from the focal point
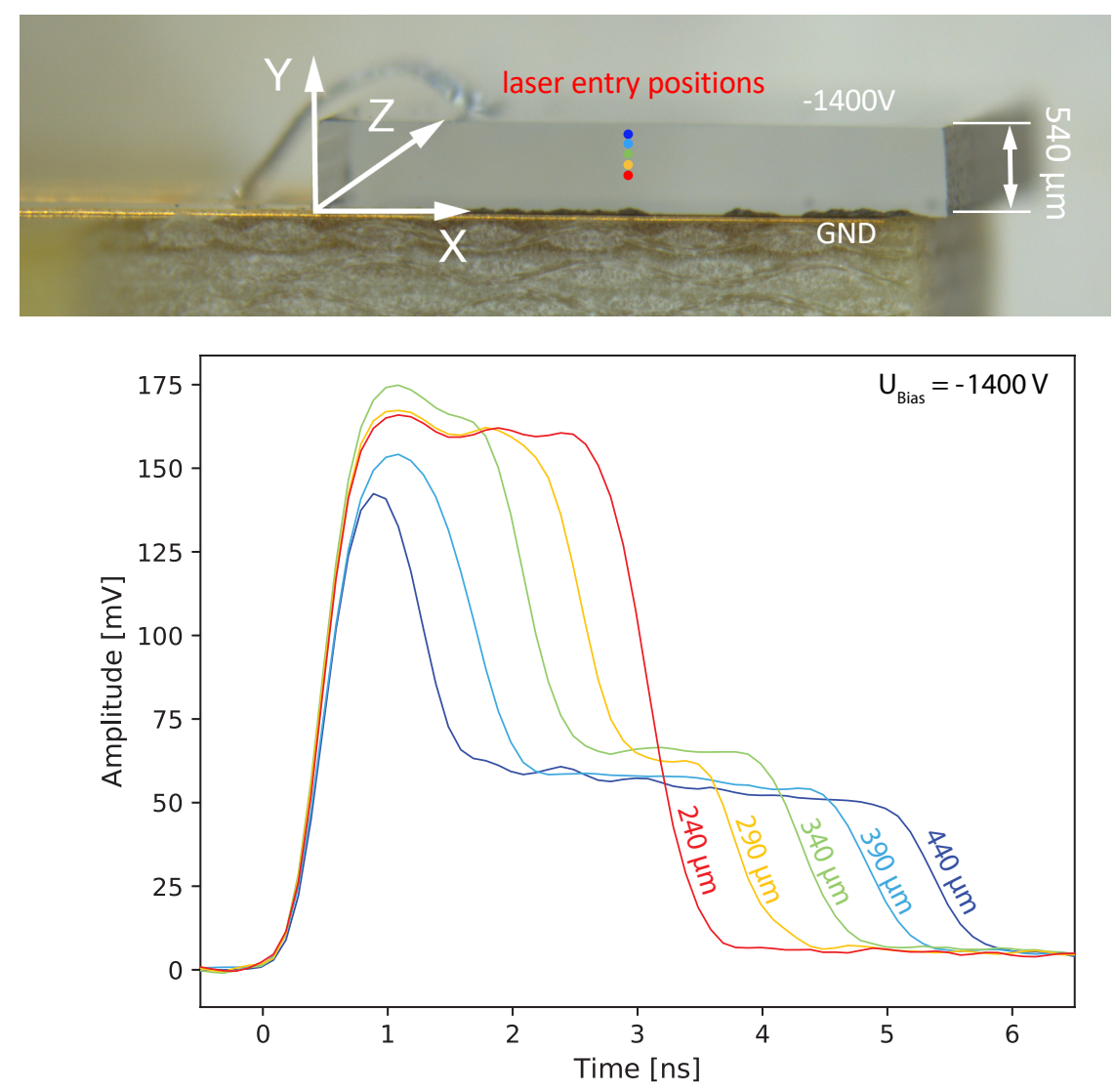

Figure 6: (Top) A side view of scCVD diamond in edge-TCT system. (Bottom) A sample edgeTCT pulse at $\mathrm{X}=\mathrm{Z}=$ and several $\mathrm{Y}$ positions of the focal point inside the diamond. The $\mathrm{X}$ and $\mathrm{Y}$ positions of the focal point are marked approximately with a correspondingly colored dot in the top Figure.

close to the top electrode, consist first of a combined movement of electron and holes, followed by electron only drift after the holes are collected. The current pulse in the ballistic center of the sensor (red curve at $240 \mu \mathrm{m}$ ), where the travel time of electrons and holes to the respective contact is the same, does not have a step-wise structure.

Measuring the length of the pulses and dividing it by the distance traveled by the charge carriers, one obtains the velocity of the charge carriers. After performing the measurements at different bias voltages one obtains the dependence of the drift velocity on the electric field (Figure 7). The 


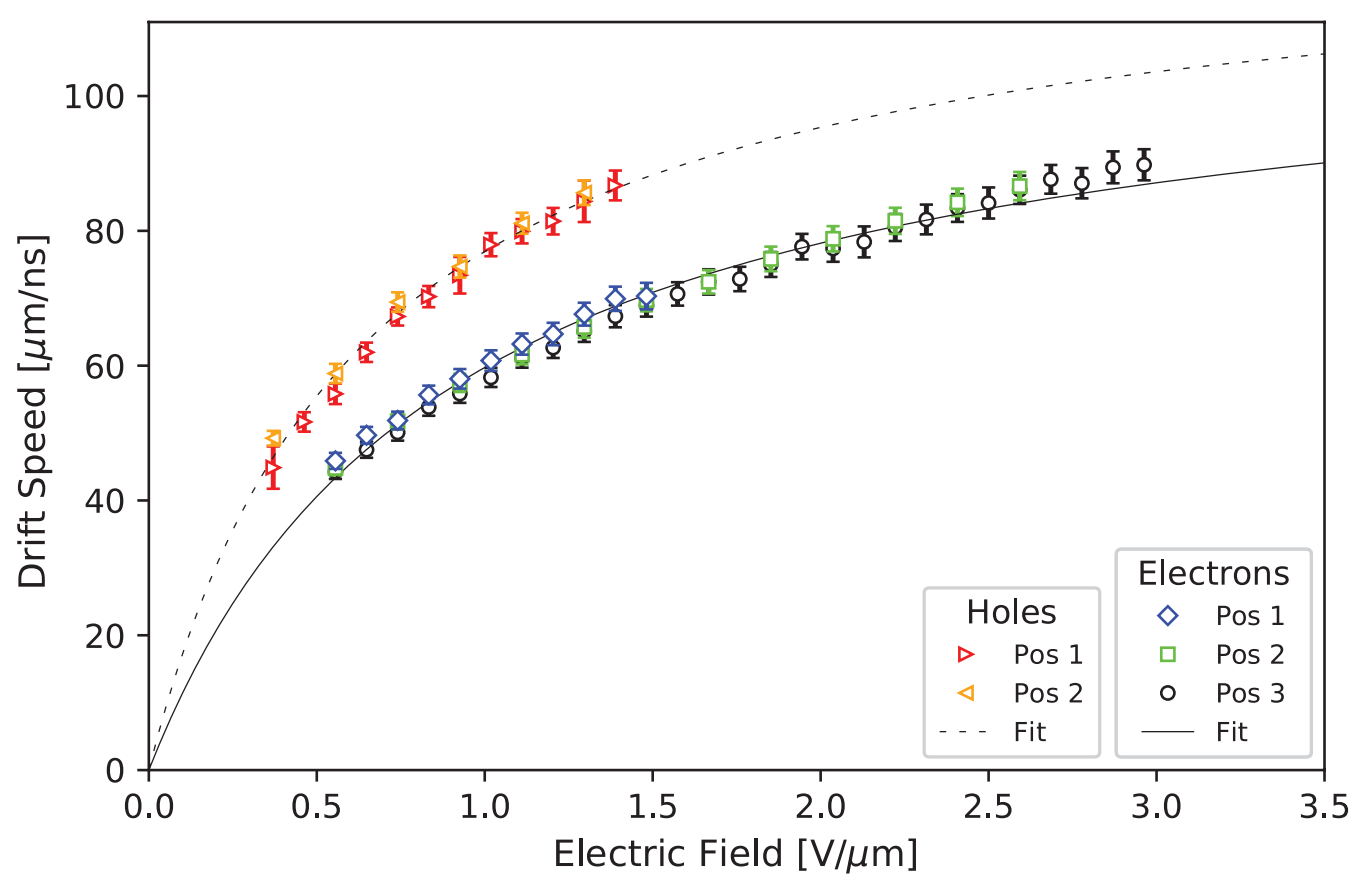

Figure 7: The drift velocity versus electric field for electrons and holes measured at several positions within the diamond. The data fitted with Equation 4.1

dependence can be fitted with an empirical relation 4.1 .

$$
v_{\text {drift }}(E)=\frac{\mu_{0} E}{1+\frac{\mu_{0} E}{v_{\text {sat }}}}
$$

The fit yields the saturation velocity and the mobility at low values of electric field (zero field mobility). For the diamond under test, the values of saturation velocity for holes and electrons respectively are: $v_{\text {sat }, h}=1.3 \times 10^{7} \mathrm{~cm} / \mathrm{s}$ and $v_{\text {sat }, e}=1.1 \times 10^{7} \mathrm{~cm} / \mathrm{s}$, while the values of the zero field mobility are: $\mu_{\text {sat }, h}=2.0 \times 10^{3} \mathrm{~cm}^{2} / \mathrm{Vs}$ and $\mu_{\text {sat }, e}=1.3 \times 10^{3} \mathrm{~cm}^{2} / \mathrm{Vs}$. These values are in the same range of values determined by other methods $[16,17]$.

By integrating the area under the current pulse (Figure 6) one can determine the total charge collected by the amplifier. Repeating this procedure for different locations of the focal point (where the charge was generated) one obtains a 3D map of charge collection efficiency. The transverse slice of that map is shown in Figure 8. One observes that for the diamond under test the charge collection is uniform with the region between the contacts and that the efficiency diminishes outside the contact area, as would be expected, because the electric field is lower there.

The electric field can also be deduced by integrating the $\sim 0.3 \mathrm{~ns}$ of the rising edge of the pulse ( $0.2 \mathrm{~ns}$ before and $0.1 \mathrm{~ns}$ after the half height of the pulse's rising edge). According to Ramo's theorem [18] this integral should be proportional to the electric field value in the immediate vicinity of the focal point. The 2D map of this integral in Figure 9 shows that the electric field is higher closer to the negative electrode, which indicates a positive space charge. It can also be noticed that the electric field change is not uniform, which indicates non-uniform distribution of the space charge, which would indicate non-uniform distribution of charge trapping defects, probably intro- 


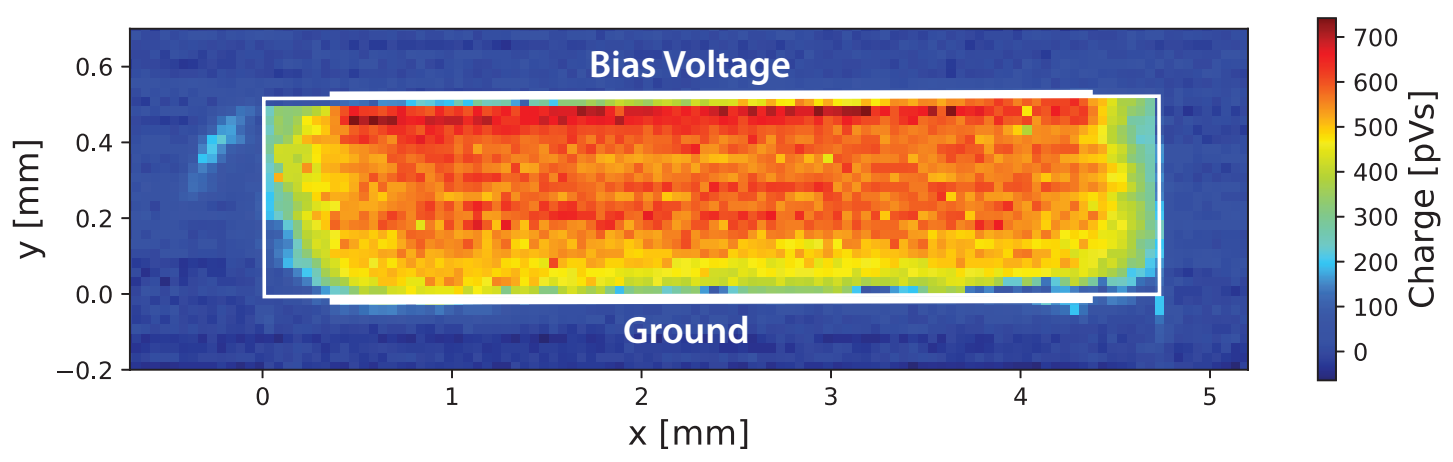

Figure 8: Collected charge versus $x-y$ position in the diamond.

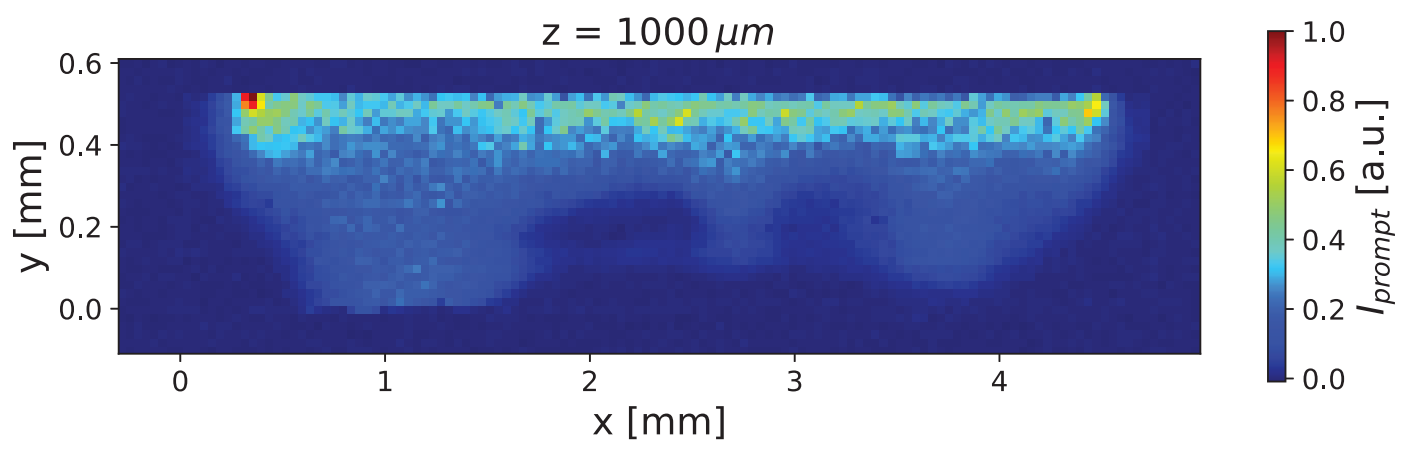

Figure 9: Prompt current profile versus x-y position in the diamond.

duced during the growth process of the diamond. The exact reason for this effect is currently under investigation.

\section{Conclusions}

We presented the latest achievements of the RD42 collaboration in the development of diamond detectors for high energy physics experiments. The updated radiation hardness coefficients of diamond are $1.04 \pm 0.07 \times 10^{-18} \mathrm{~cm}^{2} \mu \mathrm{m}^{-1}$ for $800 \mathrm{MeV}$ protons and $0.62 \pm 0.07 \times 10^{-18} \mathrm{~cm}^{2} \mu \mathrm{m}^{-1}$ for $24 \mathrm{GeV}$ protons. The radiation hardness was measured up to the fluences $2 \times 10^{16}$ protons $/ \mathrm{cm}^{2}$. Two 3D pixel detectors with $\sim 400050 \mu \mathrm{m} \times 50 \mu \mathrm{m}$ cells were fabricated with $2 \times 3$ cell ganging for PSI46digv2.1 respin chip readout and with $1 \times 5$ cell ganging for FE-I4 chip readout. Laser fabrication of $\sim 2.6 \mu \mathrm{m}$ diameter column electrodes, crucial to manufacture 3D devices, reached $99.7 \%$ efficiency. Hit detection efficiency of $>99 \%$ was achieved for the pixel detector with a 3D sensor in pCVD diamond. A two-photon edge-TCT was proven to be a useful technique to further the understanding of charge carrier transport in diamond. 3D maps of electric field in scCVD diamond can be imaged with high resolution.

\section{Acknowledgements}

The RD42 collaboration gratefully acknowledges the staff at CERN and PSI for test beam time and their help in setting up the beam conditions. The research leading to these results received 
funding from the European Union's Horizon 2020 research and innovation program under grant agreement No. 654168. This work was also partially supported by the Swiss National Science Foundation grant 20FL20 154216 and 20020 134939, ETH grant 51 15-1, Swiss Government Excellence Scholarship ESKAS No. 2015.0808, UK Science and Technology Facilities Council grant ST/M003965/1, and the U.S. Department of Energy through grant DE-SC0011726

\section{References}

[1] G. Apollinari, I. B. Alonso, O. Brüning et al., High-Luminosity Large Hadron Collider (HL-LHC): Technical Design Report V. 0.1, ser. CERN Yellow Reports: Monographs. Geneva: CERN, 2017. [Online]. Available: https://cds.cern.ch/record/2284929

[2] L. Viliani, "The cms pixel detector upgrade and r\&d developments for the high luminosity lhc," Proceedings of Science. The 26th International Workshop on Vertex Detectors (Vertex 2017) Detectors in design and Construction, vol. 309, 2018. [Online]. Available: https://doi.org/10.22323/1.309.0001

[3] M. Guthoff, W. de Boer, and S. Müller, "Simulation of beam induced lattice defects of diamond detectors using FLUKA," Nuclear Instruments and Methods in Physics Research Section A: Accelerators, Spectrometers, Detectors and Associated Equipment, vol. 735, pp. 223 - 228, 2014. [Online]. Available: http://www.sciencedirect.com/science/article/pii/S0168900213012291

[4] H. Kagan and W. Trischuk, "Development of Diamond Tracking Detectors for High Luminosity Experiments at the LHC, HL-LHC and Beyond," CERN, Geneva, Tech. Rep. CERN-LHCC-2018-015. LHCC-SR-005, May 2018. [Online]. Available: https://cds.cern.ch/record/2320382

[5] L. Bäni, A. Alexopoulos, M. Artuso et al., "A study of the radiation tolerance of poly-crystalline and single-crystalline CVD diamond to $800 \mathrm{MeV}$ and $24 \mathrm{GeV}$ protons," Journal of Physics D: Applied Physics, vol. 52, no. 46, p. 465103, aug 2019. [Online]. Available: https://doi.org/10.1088\%2F1361-6463\%2Fab37c6

[6] F. Bachmair, "CVD Diamond Sensors In Detectors For High Energy Physics," Ph.D. dissertation, Zurich, ETH, 2016. [Online]. Available: https://inspirehep.net/record/1503510/files/CERN-THESIS-2016-163.pdf

[7] L. Baeni, “Top Quarks and Diamonds,” 2017, presented 2017. [Online]. Available: https://cds.cern.ch/record/2652765

[8] S. Parker, C. Kenney, and J. Segal, "3D - A proposed new architecture for solid-state radiation detectors," Nuclear Instruments and Methods in Physics Research Section A: Accelerators, Spectrometers, Detectors and Associated Equipment, vol. 395, no. 3, pp. 328 - 343, 1997. [Online]. Available: http://www.sciencedirect.com/science/article/pii/S0168900297006943

[9] S. M. Pimenov et al., "Femtosecond laser microstructuring in the bulk of diamond," Diamond and Related Materials, vol. 18, no. 2, pp. 196 - 199, 2009. [Online]. Available: http://www.sciencedirect.com/science/article/pii/S0925963508003981

[10] M. Garcia-Sciveres, D. Arutinov, M. Barbero et al., "The FE-I4 pixel readout integrated circuit," Nuclear Instruments and Methods in Physics Research Section A: Accelerators, Spectrometers, Detectors and Associated Equipment, vol. 636, no. 1, Supplement, pp. S155 - S159, 2011, 7th International "'Hiroshima"" Symposium on the Development and Application of Semiconductor Tracking Detectors. [Online]. Available:

http://www.sciencedirect.com/science/article/pii/S0168900210009551 
[11] J. H. Hoß, "Search for Supersymmetry with Multiple Charged Leptons at sqrts = 13 TeV with CMS and Radiation Tolerance of the Readout Chip for the Phase I Upgrade of the Pixel Detector," 2017. [Online]. Available: https://cds.cern.ch/record/2287141

[12] (2019) CERN SPS H6 Beam Line. http://sba.web.cern.ch/sba/BeamsAndAreas/resultbeam.asp?beamline=H6.

[13] J. Janssen. (2020) Beam Telescope Analysis (BTA) software. https://github.com/SiLab-Bonn/beam_telescope_analysis.

[14] G. Kramberger, V. Cindro, I. Mandić et al., "Investigation of Irradiated Silicon Detectors by Edge-TCT,” IEEE Transactions on Nuclear Science, vol. 57, no. 4, pp. 2294-2302, Aug 2010.

[15] C. Dorfer, D. Hits, L. Kasmi et al., "Three-dimensional charge transport mapping by two-photon absorption edge transient-current technique in synthetic single-crystalline diamond," Applied Physics Letters, vol. 114, no. 20, p. 203504, 2019. [Online]. Available: https://doi.org/10.1063/1.5090850

[16] H. Pernegger, S. Roe, P. Weilhammer et al., "Charge-carrier properties in synthetic single-crystal diamond measured with the transient-current technique," J. Appl. Phys., vol. 97, p. 073704, 2005.

[17] M. Gabrysch, S. Majdi, D. J. Twitchen, and J. Isberg, "Electron and hole drift velocity in chemical vapor deposition diamond," J. Appl. Phys., vol. 109, p. 063719, 2011.

[18] S. Ramo, “Currents induced by electron motion,” Proc. IRE, vol. 27, p. 584, 1939. 